Textures and Microstructures, Vol. 30, pp. 207-227 Reprints available directly from the publisher Photocopying permitted by license only
(C) 1998 OPA (Overseas Publishers Association) N.V. Published by license under the Gordon and Breach Science Publishers imprint. Printed in India.

\title{
COMMENTS TO A PUBLICATION OF T.I. SAVYOLOVA CONCERNING DOMAINS OF DEPENDENCE IN POLE FIGURES
}

\author{
S. MATTHIES ${ }^{a, b, \dagger}$ and C. ESLING ${ }^{a, *}$ \\ ${ }^{a}$ Université de Metz, Laboratoire LETAM URA CNRS 2090, ISGMP, \\ Jle du Saulcy, F-57045 METZ Cedex 01, France; \\ ${ }^{b}$ Seoul National University, School of Materials Science \& Engineering, \\ Seoul 151-742, South Korea
}

(Received 12 July 1996; In final form 5 December 1997)

A questionable publication of T.I. Savyolova is analysed. The paper claims, that it is possible to determine $\boldsymbol{P}_{\mathrm{h}}(\mathrm{y})$ pole figure values for $\mathrm{y} \in \boldsymbol{Y}\left(\mathbf{h} ; \mathbf{h}_{\mathbf{0}}, \boldsymbol{Y}_{\mathbf{0}}\right)$ using only data from $\mathrm{y} \in Y_{0}$ of a single pole figure $P_{\mathrm{h}_{0}}(\mathrm{y})$. The contradiction to the common knowledge can be resolved well understanding that there is a general difference between the term solutions (or continuation of solutions) of an ultrahyperbolic equation (satisfied by the axis distribution function $\boldsymbol{A ( h , y ) )}$ and the term pole figure. Pole figures considered in texture analysis are two-dimensional projections of a three-dimensional object (the ODF $f(g)$ ). For limited data sets the equation $\Delta_{\mathrm{h}} A(\mathbf{h}, \mathbf{y})=\Delta_{\mathbf{y}} A(\mathbf{h}, \mathbf{y})$ bears only a necessary, but not sufficient character in order to get solutions of interest, i.e. it cannot be guaranteed that a h-specific continuation of a starting solution $\boldsymbol{P}_{\mathrm{h}_{0}}(\mathbf{y})$ will be a "h-projection" of the same ODF, which belongs to a concrete sample and possesses the "h$h_{0}$-projection" $\boldsymbol{P}_{\mathbf{h}_{0}}(\mathbf{y})$.

Consequently, to name such a h-specific continuation "pole figure" is incorrect. The consideration of formal (unambiguous only by artificial conditions) continuations of solutions may be mathematically interesting, but is of no practical importance for texture analysis. Examples (already considered by the authors about twenty years ago) are given, how to construct in a much more simple way continuations of solutions of the same useless type like in the paper under discussion.

Keywords: Pole figures; Domains of dependence; Ultra-hyperbolic differential equation; Continuation of solutions

* Corresponding author.

${ }^{\dagger}$ Permanent address: Mueller-Berset-Str. 3, D-1309 Dresden, Germany. 


\section{INTRODUCTION}

In her publication "Calculation of domains of dependence for pole figures with an ultrahyperbolic equation" (Savyolova, 1995; see also Savyolova, 1996) the author comes to the spectacular conclusion that it is possible, using the (arbitrary) intensity distribution given in a $\mathbf{y}$ region $Y_{0}$ of a single pole figure $P_{\mathrm{h}_{0}}(\mathbf{y})$, to determine the intensities for some other pole figures $P_{\mathbf{h}_{i}}(\mathbf{y})$ in $\left(\mathbf{h}_{0}, Y_{0}, \mathbf{h}_{i}\right)$-specific regions $Y_{i}$.

We definitely disagree with this statement.

The reason for the dubious conclusion by T.I. Savyolova is finally a misunderstanding and not appropriate use of the (well defined in texture analysis) term "pole figure", considering solutions of an equation of only necessary but not sufficient character. The mathematically formulated reply can therefore be relatively short and is given in the appendix.

There are some motives for an enlarged form of the present publication:

- The "fairy-tale" on ODF-determinations, using only one (and even incomplete) pole figure still appears from time to time in literature.

- We would like to recommend potentially interested readers of Savyolova's paper not to waste time in a, at first sight, new interesting direction (cf. Nikolayev and Schaeben, 1997).

And at last:

- It is not simple to read and to analyse Savyolovas paper (doubtless containing interesting valuable mathematical aspects) and to find out the critical moments related to texture analysis.

Therefore (unlike the request of the appendix) in the main part we try to give some necessary background information, more or less popular (or even simplified) explanations of the nontrivial interesting problem, and will demonstrate several weak points of the discussed paper.

Also, because Savyolova's idea, if it works, must be applicable to the case of "ideal pole figures" too (triclinic (i.e. "no") crystal symmetry and no $\pm \mathbf{h}_{\boldsymbol{i}}$-reduction by Friedels law), at the beginning we will not consider these complicating features, but unessential for the basic problem under consideration. 


\section{THE EXACT SOLUTION OF THE CENTRAL PROBLEM OF TEXTURE ANALYSIS}

We have already seen in the past, that there may appear some difficulties between abstract mathematical considerations of a problem and its realization in a "language" prescribed by the experimental possibilities, i.e. adapted to the practical situation in texture analysis.

So, at the very beginning of the 1980 s, we derived, for instance, the mathematically exact solution of the problem of pole figure inversion in a closed analytical form using two completely different methods (Matthies, 1979; 1980; Muller et al., 1981). For the above mentioned ideal case ("ideal pole figures" $P$ ) this solution reads:

$$
\begin{aligned}
f(g)= & \int_{H} f(\mathbf{h} ; g) \mathrm{d} \mathbf{h} / 4 \pi, \\
f(\mathbf{h} ; g)= & P_{\mathbf{h}}\left(g^{-1} \cdot \mathbf{h}\right) \\
& +\int_{0}^{\pi}\left[2 \pi P_{\mathbf{h}}\left(g^{-1} \cdot \mathbf{h}\right)-W\left(\cos \vartheta^{\prime}, \mathbf{h}, g\right)\right] \frac{\cos \vartheta^{\prime} / 2}{\sin ^{2} \vartheta^{\prime} / 2} \mathrm{~d} \vartheta^{\prime} / 4 \pi \\
\equiv & f_{1}(\mathbf{h} ; g)+f_{2}(\mathbf{h} ; g),
\end{aligned}
$$

with

$$
W\left(\cos \vartheta^{\prime}, \mathbf{h}, g\right)=\int_{0}^{2 \pi} P_{\mathbf{h}}\left(\left\{g^{-1} \cdot \mathbf{h}, 0\right\}^{-1} \cdot \mathbf{y}^{\prime}\right) \mathrm{d} \varphi^{\prime} ; \quad \mathbf{y}^{\prime}=\left(\vartheta^{\prime}, \varphi^{\prime}\right)
$$

Two aspects are to be underlined in this connection. First, only pole figures are asked explicitly as entry data of the solution. Second, the exact solution requires the complete pole figures (all sample directions y) and those for all crystal directions $h$. This cannot be achieved in practice, because, among other difficulties, diffraction peaks are available only in the form of finite discrete $\mathbf{h}_{i}$-sets.

Therefore, the aim of any method of pole figure inversion is to extract maximum information about the ODF from the deficient 
volume of experimental data. In this respect, reasonable ODF approximations ought to be constructed, and the restricted nature of the pole figure-related input data should concern only the resolution power of these approximations.

From a mathematical point of view, there is a perfect symmetry between the direct $P_{\mathrm{h}}(\mathrm{y})$ and the inverse $R_{\mathrm{y}}(\mathrm{h})$ pole figures which can both be considered as specific representations of the so-called biaxial function $A(\mathbf{h}, \mathbf{y})$ (Bunge, 1982):

$$
P_{\mathbf{h}}(\mathbf{y})=R_{\mathbf{y}}(\mathbf{h})=A(\mathbf{h}, \mathbf{y})=\int_{0}^{2 \pi} f\left(\{\mathbf{h}, \tilde{\varphi}\}^{-1} \cdot\{\mathbf{y}, 0\}\right) \mathrm{d} \tilde{\varphi} / 2 \pi .
$$

Disregarding problems of experimental feasibility and considering $A(\mathbf{h}, \mathbf{y})$ as a purely mathematical object with continuously varying $\mathbf{y}$ and $\mathbf{h}$, abstract coordinate transformations mixing $\mathbf{h}$ and $\mathbf{y}$ are also imaginable.

In 1982, Savyolova published a rather elaborate study from a mathematical point of view, that was indeed based on such mixed variables. We could infer from this paper that the problem of pole figure inversion in such a representation can be solved using the socalled Radon transformation and that there is an infinite number of possible formulations of the solution of the problem. However, up to now there has been published not even one variant from this multiplicity of solutions, which refers to experimentally accessible quantities in terms of $h$ and $y$ and which can be directly compared with our simple, analytically closed and transparent solution (Eqs. (1), (2)).

\section{ANALYTICAL DEPENDENCIES BETWEEN POLE FIGURES}

A quite simple fact immediately leads to acknowledging that there must be some kind of dependency between $y$ and $h$ in the biaxial function $A(\mathbf{h}, \mathbf{y})$ (cf. Eq. (3)). As is well known, the orientation space $G$ has a volume of $8 \pi^{2}$ :

$$
\int_{G} \mathrm{~d} g=\int_{0}^{2 \pi} \mathrm{d} \alpha \int_{0}^{2 \pi} \mathrm{d} \gamma \int_{0}^{\pi} \sin \beta \mathrm{d} \beta=8 \pi^{2} ; \quad g=\{\alpha, \beta, \gamma\}
$$


Consequently, a "volume of information" of $8 \pi^{2}$ should be sufficient for the reconstruction of the ODF. But the solution of Eqs. (1), (2) requires a twice larger volume $16 \pi^{2}$ :

$$
\left(\int_{H} \mathrm{dh}=4 \pi\right) \cdot\left(\int_{Y} \mathrm{dy}=4 \pi\right)=16 \pi^{2} .
$$

In other words, to obtain the three-dimensional information $f(\{\alpha, \beta, \gamma\})$, a four-dimensional data set $A\left(\vartheta_{\mathbf{h}}, \varphi_{\mathbf{h}} ; \vartheta_{\mathbf{y}}, \varphi_{\mathbf{y}}\right)$ is required. This contradiction can be reconciled when we interpret the above factor 2 as the price we have to pay insisting on $\mathbf{h}$ and $\mathbf{y}$ as variables of the entry quantities (pole figures) of the reconstruction problem (because only these are experimentally available) supposing, however, that an $\mathbf{h} \leftrightarrow \mathbf{y}$ dependency can be found in each point $(\mathbf{h}, \mathbf{y})$ for $A(\mathbf{h}, \mathbf{y})$. This dependency is quite simple and reads:

$$
\Delta_{\mathbf{h}} A(\mathbf{h}, \mathbf{y})=\Delta_{\mathbf{y}} A(\mathbf{h}, \mathbf{y}),
$$

where $\mathbf{r} \equiv(\vartheta, \varphi)$, and $\Delta_{\mathbf{r}}$ is the angular part of the Laplace operator in spherical co-ordinates:

$$
\Delta_{\mathbf{r}}=\left(1-z^{2}\right) \frac{\partial^{2}}{\partial z^{2}}-2 z \frac{\partial}{\partial z}+\frac{1}{1-z^{2}} \frac{\partial^{2}}{\partial \varphi^{2}}, \quad z=\cos \vartheta
$$

The harmonic method is based on the eigenfunctions $Y_{l, m}(\mathbf{r})$ of this operator (Edmonds, 1957) with the eigenvalues $l(l+1)$ :

$$
\Delta_{\mathbf{r}} Y_{l, m}(\mathbf{r})=l(l+1) Y_{l, m}(\mathbf{r}) .
$$

The series representation of pole figures by these eigenfunctions is absolutely model-independent:

$$
A(\mathbf{h}, \mathbf{y})=4 \pi \sum_{l=0}^{\infty} \sum_{m, n=-l}^{l} \frac{1}{2 l+1} C_{l}^{m, n} Y_{l, m}^{*}(\mathbf{h}) Y_{l, n}(\mathbf{y}) .
$$

Using (8), Eq. (6) can immediately be deduced from (9). The relation (6) has already been observed and discussed by us during international methodological seminars in Metz in the early 1980s (Baro et al., 1982). 
It was analysed at that time as a possibility to reduce the necessary volume of experimental data for ODF reconstruction. But finally it was rejected.

The reason why we have concluded that this path is an impasse is the following. Equation (6) obviously connects the $y$ and $h$ spaces in a specific way. Thus, as a matter of principle, it should be possible to transform y-related information into a h-related one. Since Eq. (6) (using the most abstract characterization) is a partial second-order differential equation for a function of four variables, an unique solution requires information on the values of the asked function (as well as on its first derivatives in $\mathbf{h}$ ) on a finite $\mathbf{h}$-depending three-dimensional boundary, i.e. in any case, these requirements will be linked with continuous $h$ variations, which are mathematically conceivable, but cannot be realized experimentally (not even in a bad approximation, especially for the derivatives, due to the discrete and accidental character of the available reflections $\mathbf{h}_{i}$ ).

At least from this formal view, it seems apparent to us, that one single pole figure (a single $h$, i.e. one point in the $h$ space) cannot serve as a basis for reasonable estimations of distributions in a continuous $h$ domain.

Solutions of Eq. (6) of common (ambiguous) character, or formally constructed in an unique way, but using artificial model conditions are not of practical interest. Moreover, up to now we even have not considered the much more important question, whether Eq. (6) will be able at all to deliver solutions of interest for texture analysis resting on a limited set of information only.

\section{CONTINUATION OF SOLUTIONS}

At first sight, it seems possible to transfer information from data of a $\left(h_{0}\right)$ pole figure measured in the domain $Y_{0}$ to another $\left(h_{1}\right)$ pole figure at the expense of the size of the $Y_{1}$ domain $\left(Y_{1}<Y_{0}\right)$. Representing schematically the projection character of pole figures as in Fig. 1 (in the real case the projection lines in the $G$ space have identical lengths), then for the $Y_{1}$ domain of $P_{\mathrm{h}_{1}}(\mathbf{y})$ only an ODF information from the $A$ domain is necessary, that is contained in $P_{\mathrm{h}_{0}}(\mathrm{y})$ in the $Y_{0}$ domain, in the form of an integrated information. 


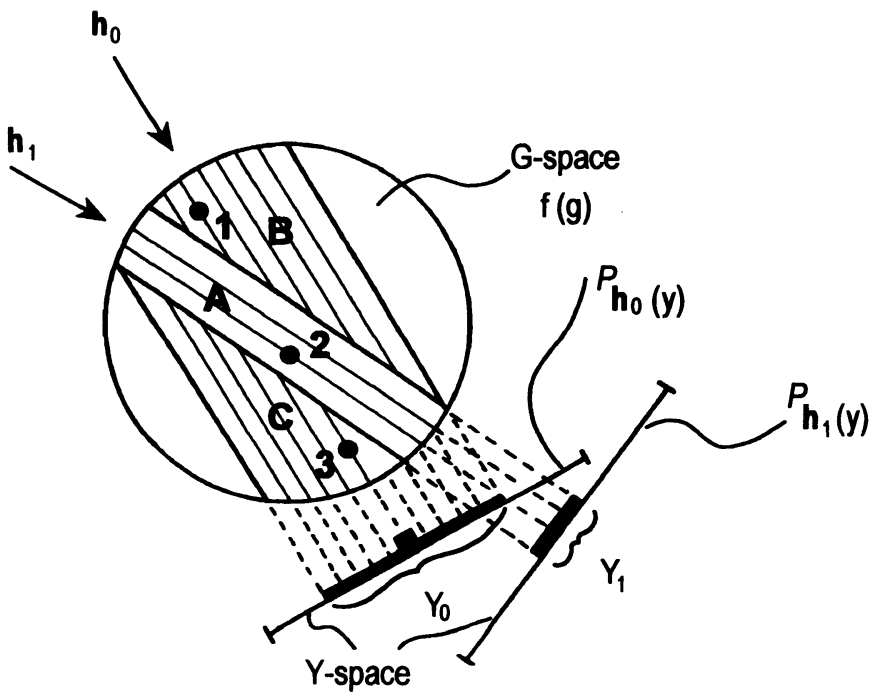

FIGURE 1 Schematic illustration of the projection character of pole figures.

But the question is, whether this information can be separated from the superimposed contributions coming from $B$ and $C$. Disposing of some pole figures, whose $\mathbf{h}_{i}$ continuously (or in small steps) vary from $\mathbf{h}_{\mathbf{0}}$ towards $\mathbf{h}_{\mathbf{1}}$, a solution is conceivable. However, with one single pole figure, in spite of the support provided by Eq. (6), this is impossible because the ODF values in the domains $B$ and $C$ can be chosen freely within a wide variation range, always preserving the same $P_{\mathrm{h}_{0}}(\mathbf{y})$.

If a maximum of intensity is observed in $P_{\mathrm{h}_{0}}(\mathbf{y})$, it can originate from distributions along the whole corresponding projection path (i.e. for instance, from the "texture components" 1,2 or 3). Considering now $\boldsymbol{P}_{\mathrm{h}_{1}}(\mathbf{y})$, depending on whether " 2 " is absent or not, we will have a lack of intensity in $Y_{1}$, or an intensity can appear in $Y_{1}$ at arbitrary positions $\mathbf{y}_{1}$, without any changes in $P_{\mathrm{h}_{0}}(\mathbf{y})$.

Consequently, when it is claimed that $P_{\mathrm{h}_{1}}(\mathrm{y})$ data in $Y_{1}$ can be explained with the help of $P_{\mathrm{h}_{0}}(\mathrm{y})$ data from $Y_{0}$ only, it should be possible to construct convincing examples of the above type, which are sufficient as counter-evidence. Of course, obvious errors in demonstrations or results are also informative about the quality of the treatment of the theme. 
Some decisive circumstances have to be underlined yet. Independently from the fact whether at all - and how - an information can be transmitted from $Y_{0}$ to $Y_{1}$, at least one thing is quite sure: the domain $Y_{1}$ for $P_{\mathrm{h}_{1}}(\mathrm{y})$ is determined through $A$ purely geometrically by $\mathrm{h}_{0}, \mathrm{~h}_{1}$ and $Y_{0}$. Concerning its determination, equations of type (6) are absolutely unimportant. On the contrary, possible formal continuations of solutions into a domain $Y_{1}^{*}$, which are deduced from an abstract treatment of Eq. (6), must be complemented with additional conditions such as $Y_{1}^{*} \cap Y_{1}$. This is the minimum level at which the necessary information, according to which the $P_{\mathrm{h}}(\mathbf{y})$ are projections of a common object defined in a space of higher dimension, has to be taken into account. This common property of true pole figures in no way follows from Eq. (6) or any transformed variants of it without additional information.

Indeed, only possessing the data of $A(\mathrm{~h}, \mathrm{y})$ for all $\mathrm{h}$ and $\mathrm{y}$, where all are satisfying (6), the projection character in the form (3) can be concluded for this data set. Equation (6) does not know anything whatsoever about projections.

In the most general solution - Eq. (9) - arbitrary $C_{l}^{m, n}$ values are admissible. For a $C_{l, n}^{m, n}$ set, that exactly explains $P_{\mathrm{h}_{0}}(\mathbf{y}) \geq 0$ (in the common case the $C_{l}^{m, n}$ cannot be determined unambiguously from one given pole figure, see also Bunge and Esling, 1979), even the fulfillment of the simple condition $P_{\mathbf{h}_{1}}(\mathbf{y}) \geq 0$ cannot be guaranteed for a " $h_{1}$ pole figure" formally calculated via Eq. (9), although it is an exact solution of Eq. (6).

In other words, Eq. (6) is only a necessary equation that real pole figures (or more exactly $\rightarrow$ biaxial distributions) have to fulfil. But Eq. (6) is not sufficient to deliver true pole figure data $P_{\mathrm{h}_{1}}(\mathbf{y})$ by any kind of "continuation of its solutions", resting only on $P_{\mathrm{h}_{0}}(\mathbf{y})$-data.

Therefore, the contradiction under discussion can be resolved well understanding that there is a general difference between the terms "solutions or continuations of solutions" of Eq. (6) (that might be of some abstract mathematical interest) and the term "pole figure" in the sense (3), the only object of interest for texture analysis. There is nothing new about the determination of the $Y_{i}$ either. Since the mid 1970s (beginning with the vector method (Ruer and Baro, 1977)) its inverse formulation (which $A$ - cf. Fig. 1 - do result from $h_{0}, Y_{0}, h_{1}, Y_{1}$ ?) is a part of all computer programs using direct ODF reconstruction 
methods. As to this subject, refer also to the extensive investigations by Vadon (1981) about the MPDS concept.

\section{CONCERNING THE PAPER BY T.I. SAVYOLOVA}

In spite of the possibility to demonstrate the main idea considering first the simplest case of ideal pole figures, Eq. (3) (without symmetries and loss of information in the normal diffraction experiment (Friedel's law)), her paper is burdened with all these elements of secondary importance for the problem under consideration. As a consequence it is rather difficult to follow it up and to analyse it in detail. The basic program of the author is as follows. By transforming variables $h$ and $\mathbf{y}$ (10), it is possible to bring Eq. (6) $(A \rightarrow F)$ into the form of an ultrahyperbolic Eq. (11):

$$
\begin{gathered}
x_{1}=x_{1}(\mathbf{h}, \mathbf{y}), \quad x_{2}=x_{2}(\mathbf{h}, \mathbf{y}), \quad z_{1}=z_{1}(\mathbf{h}, \mathbf{y}) \quad \text { and } \quad z_{2}=z_{2}(\mathbf{h}, \mathbf{y}), \\
\frac{\partial^{2} F\left(x_{1}, x_{2}, z_{1}, z_{2}\right)}{\partial x_{1}^{2}}+\frac{\partial^{2} F\left(x_{1}, x_{2}, z_{1}, z_{2}\right)}{\partial x_{2}^{2}} \\
=\frac{\partial^{2} F\left(x_{1}, x_{2}, z_{1}, z_{2}\right)}{\partial z_{1}^{2}}+\frac{\partial^{2} F\left(x_{1}, x_{2}, z_{1}, z_{2}\right)}{\partial z_{2}^{2}}
\end{gathered}
$$

Then $x_{1}$ and $x_{2}$ are transformed into polar coordinates $(r, \varphi)$ and $z_{1}$ and $z_{2}$ into $(\rho, \phi)$, respectively. Considering $F$ as an abstract function of these variables it can be decomposed into a series of Bessel functions similar to Eq. (9). In this respect, a model parameter $a>0$ is required and can either be finite or infinite. For known solutions in given $r$ and $\rho$ domains there are in both cases possibilities to continue the solution into new regions of the $\rho$ domain, correspondingly lowering the size of the $r$ domain. Some prescriptions for the determination of the Bessel function series coefficients are also provided (S. (11)). Analysing these connections in more detail the necessity of the above-mentioned $\mathbf{h}$ derivations, when we analysed in Section 3 Eq. (6) as a partial secondorder differential equation, can be seen. 
Then an attempt is made to apply the results of the abstract case to the case of pole figures (i.e. $F=A$ ). Concretely, the case of a single pole figure and only for special h with $\vartheta_{\mathrm{h}}=\pi / 2$ is considered.

However, as opposed to the above abstract case, the description of the ways to get new solutions is relatively fuzzy and difficult to follow. For instance, the series coefficients have to be derived from a system of algebraic equations (see below S. (17)) which is apparently underdetermined in the general case (this is for example in agreement with the difficulties mentioned in connection with the analysis of Fig. 1).

The consequence of the forced coupling of $\mathbf{h} \leftrightarrow \mathbf{y}$ in the Bessel functions (instead of being clearly separated as in the exact expression (9)) in order to get a resolvable scheme are assumptions of kind $F(\rho=a)=0$, or $\partial F /\left.\partial \rho\right|_{\rho=a}=0$. After this, the ambiguity of a continued solution may be overcome. However, these conditions having been imposed in an artificial way, they may lead to satisfactory approximations for an infinite space, but they may also have consequences in the finite pole figure space of a magnitude that can hardly be evaluated.

Furthermore, an independence of $F$ from $\varphi_{\mathbf{h}}$ is mentioned. This does not correspond to the reality (apart from a trivial particular case - see below), because crystal lattices with cylindrical symmetry do not exist (which additionally was supposed for any $h$ ).

After assuming that the solutions in the domain of continuation can be determined on the basis of the data of one pole figure with $\vartheta_{\mathrm{h}_{0}}=\pi / 2$ (cf. S.p. 190), and using the conditions for the continuation of solutions of Eq. (11), concrete formulas for $\mathbf{y}$ domains $Y_{i}^{*}$ in other $\mathbf{h}_{i}$ pole figures are given as a function of the measured $y$, i.e. of the domain $Y_{0}$ in the $h_{0}$ pole figure.

Trying to confirm the results obtained by the author, at least for some numerical examples, we already failed with the very first continuation domain. Using the relations at the bottom of S. p. 190 from $a=2(2+\sqrt{3})$, it follows $\chi_{l}=60^{\circ}$. For the $h_{0}$ of the pole figure measured in the domain $Y_{0}\left(0 \leq \vartheta_{y_{0}} \leq 60^{\circ}, 120^{\circ} \leq \vartheta y_{0} \leq 180^{\circ}\right)$ we select $\vartheta_{\mathrm{h}_{0}}=90^{\circ}, \varphi_{\mathrm{h}_{0}}=100^{\circ}$.

A texture component in the $G$ space at $g=\{\alpha, \beta, \gamma\}=\left\{0^{\circ}, 105^{\circ}, 0^{\circ}\right\}$ yields a peak in $P_{\mathbf{h}_{0}}(\mathbf{y})$ at $\mathbf{y} \equiv(\vartheta, \varphi)=g^{-1} \cdot \mathbf{h}_{0}$. With

$g^{-1}=\{\pi-\gamma, \beta, \pi-\alpha\} \quad$ and $\quad \mathbf{h}_{\mathbf{0}}=\left(\vartheta_{\mathbf{h}_{0}}, \varphi_{\mathbf{h}_{0}}\right) \equiv\left\{\varphi_{\mathbf{h}_{0}}, \vartheta_{\mathbf{h}_{0}}, 0\right\}^{-1}\left(0^{\circ}, 0^{\circ}\right)$ 
it follows immediately that

$$
\begin{aligned}
\mathbf{y} & =\{\pi, 0,0\}\left\{\pi, 105^{\circ}, 0\right\}\left\{\pi, 90^{\circ}, \pi-100^{\circ}\right\}\left(0^{\circ}, 0^{\circ}\right) \\
& \equiv\{\pi, 0,0\}\left\{\alpha^{*}, \beta^{*}, \gamma^{*}\right\}\{\pi, 0,0\}\left(0^{\circ}, 0^{\circ}\right) \\
& =\left\{\alpha^{*}+\pi, \beta^{*}, \gamma^{*}+\pi\right\}\left(0^{\circ}, 0^{\circ}\right)=\left\{-\gamma^{*}, \beta^{*},-\alpha^{*}\right\}^{-1}\left(0^{\circ}, 0^{\circ}\right) \\
& =\left(\vartheta_{\mathbf{y}}=\beta^{*}, \varphi_{\mathbf{y}}=-\gamma^{*}\right),
\end{aligned}
$$

with

$$
\left\{\alpha^{*}, \beta^{*}, \gamma^{*}\right\}=\left\{-100^{\circ}, 105^{\circ}, 0\right\}\left\{0,90^{\circ}, 0\right\} \equiv\left\{\varepsilon, \beta_{2}, 0\right\}\left\{0, \beta_{1}, 0\right\}
$$

For $\beta^{*}$, the elementary equation $\cos \beta^{*}=\cos \beta_{1} \cos \beta_{2}-\sin \beta_{1} \sin \beta_{2} \times$ $\cos \varepsilon$ is valid and we get $\beta^{*}=80.34^{\circ}$, i.e. the pole figure $P_{\mathrm{h}_{0}}(\mathbf{y})$ is empty in the measured domain $Y_{0}$ (see above).

According to S.p. 191 (top), $\theta^{*}=30^{\circ}$ applies for $a=2(2+\sqrt{3})$. According to point la (cf. S.p. 191) all pole figures with $60^{\circ} \leq$ $\vartheta_{\mathbf{h}_{i}} \leq 90^{\circ}$ can be determined in the $Y_{i}^{*}$ domains $(\pi / 2)-\vartheta_{\mathbf{h}_{i}} \leq \vartheta_{\mathbf{y}} \leq$ $-(\pi / 2)+\vartheta_{\mathbf{h}_{i}}+2 \theta^{*}$ on the basis of the $P_{\mathbf{h}_{0}}(\mathbf{y})$ pole figure. We select $\vartheta_{\mathbf{h}_{1}}=75^{\circ}$ and $\varphi_{\mathbf{h}_{1}}=180^{\circ}$. This means, that this pole figure is determinable in the $Y_{1}^{*}$ domain $15^{\circ} \leq \vartheta_{\mathbf{y}} \leq 45^{\circ}$. A calculation similar to the above one immediately yields that the $g$-peak is located in this pole figure at $\vartheta_{\mathbf{y}}=30^{\circ}$, i.e. just in the centre of the "determinable" domain $Y_{1}^{*}$. Figures 2 and 3 illustrate the situation.

We have some difficulties to imagine how the author intends to generate a peak in $Y_{1}^{*}$ from the zero intensity in $Y_{0}$. Using the terminology of Fig. 1, the present case obviously belongs to a situation for which $Y_{1}^{*}$ is located outside $Y_{1}$ and possesses projection threads nowhere intersecting the $G$-space region $(A+B+C)$ determined by $Y_{0}$.

Figures 4 and 5 give an additional example in connection with the discussion of Fig. 1. If instead of the texture component at the above $g$ we have one at $g^{*}=\left\{351.85^{\circ}, 60.47^{\circ}, 1.12^{\circ}\right\}$ the $h_{0}$ pole figure will be identically the same. However, the corresponding $\mathbf{h}_{1}$ figure ("determinable" by these $h_{0}$ pole figure data) given in Fig. 5 differs from that of Fig. 3.

Though commonly one counter-example is sufficient in order to question the merit of a certain concept, it seems reasonable additionally to consider some other issues of Savyolova's paper. 


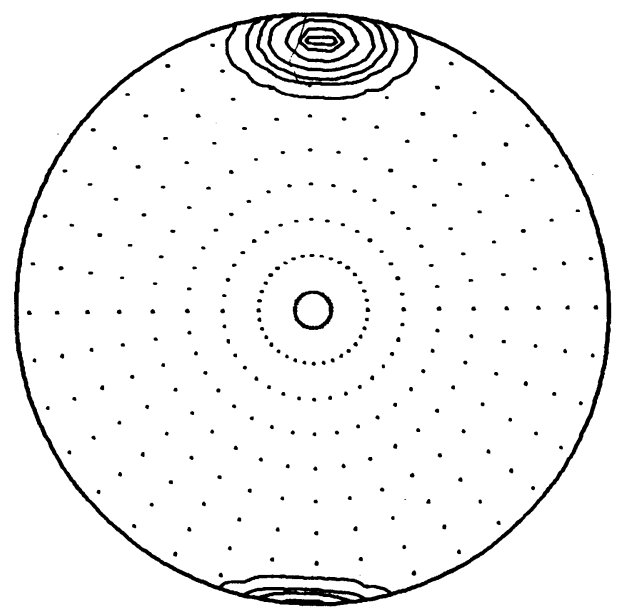

FIGURE 2 The $h_{0}=\left(\vartheta_{h}=90^{\circ}, \varphi_{h}=100^{\circ}\right)$ pole figure for one texture component (Standard Gaussian, $b=15^{\circ}$, triclinic/triclinic symmetry), located at $g=\left\{0^{\circ}, 105 ; 0^{\circ}\right\}$. The "measured" domain $Y_{0}\left(0 \leq \vartheta_{\mathrm{y}} \leq 60^{\circ}\right)$ is empty. Maximum: 80.1; isolines: 1,5, 10, $20,40,60,70,80$.

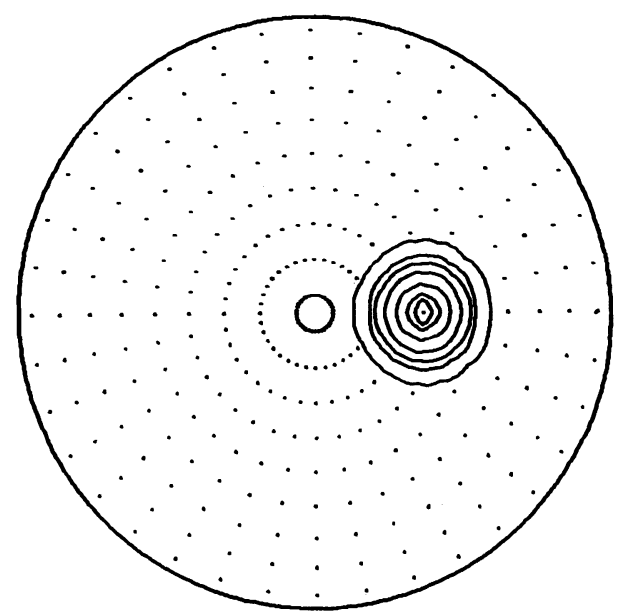

FIGURE 3 The $h_{1}=\left(\vartheta_{h}=75^{\circ}, \varphi_{h}=190^{\circ}\right)$ pole figure to be explained using the data from the $Y_{0}$ region of the $h_{0}$ pole figure given in Fig. 2. The "explainable" domain $Y_{1}^{*}:\left(15^{\circ}<\vartheta_{\mathbf{y}}<45^{\circ}\right)$ contains a peak, located at $\vartheta_{\mathbf{y}}=30^{\circ}$. 


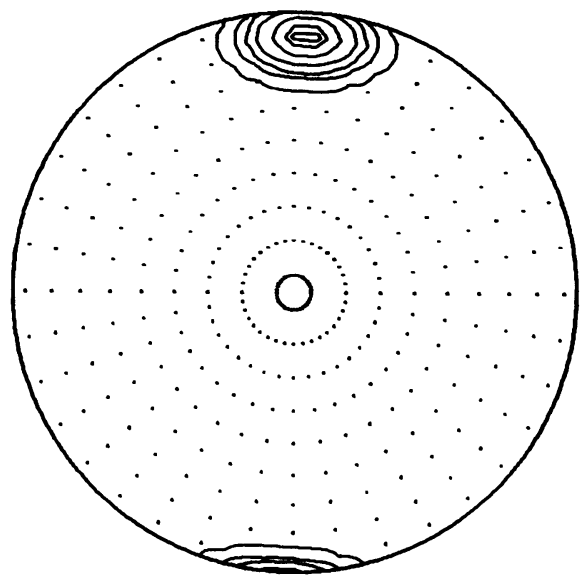

FIGURE 4 The $h_{0}=\left(\vartheta_{h}=90^{\circ}, \varphi_{h}=100^{\circ}\right)$ pole figure for a texture component located at $g^{*}=\left\{351.85^{\circ}, 60.47^{\circ}, 1.12^{\circ}\right\}$. The $h_{0}$ figure is identical with that of Fig. 2 .

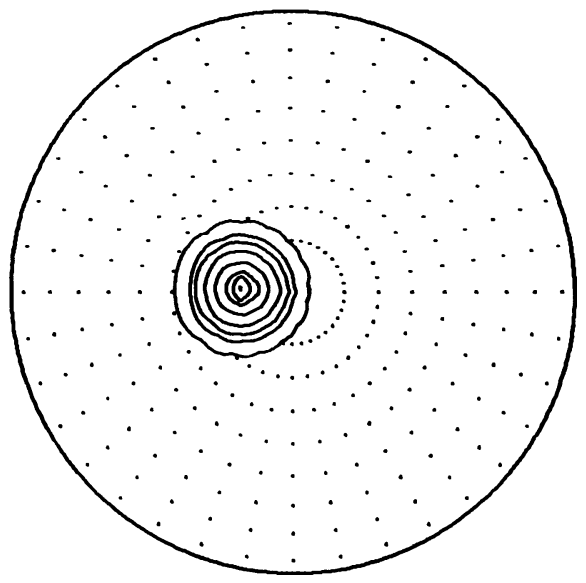

FIGURE 5 The $h_{1}=\left(\vartheta_{h}=75^{\circ}, \varphi_{h}=190^{\circ}\right)$ pole figure for the $g^{*}$ component. $P_{h_{1}}(y)$ is different from that of the $g$ component shown in Fig. 3.

\section{THE BERYLLIUM EXAMPLE}

The author tries to confirm the derived relationships on the basis of some experimental data. Unfortunately, this example only represents a very particular and irrelevant case. On the basis of the assumptions 
made, according to which $F=\boldsymbol{A}\left(\mathbf{h}_{i}, \mathbf{y}\right)$ should not depend on $\varphi_{\mathbf{h}_{i}}$ and $\varphi_{\mathbf{y}}$, it can immediately be inferred that this is possible only for a cyclic fibre component, with $\mathbf{N}=\mathbf{H}=\left(0^{\circ}, 0^{\circ}\right)$ (Matthies et al., 1990). Consequently, already the starting assumptions determine an ODF, not only a pole figure type. In other words, in Eq. (9), all $C_{l}^{m, n}$ with $m$, $n \neq 0$ have to be identical to 0 . The consequence is, that all coefficients $C_{l}^{0,0}$ completely describing the ODF in this case can be determined on the basis of only one pole figure. Thus, any single pole figure already represents the whole ODF of such a sample. But, as it is well known, any other pole figure can then be determined in the entire $y$ domain from the ODF.

In this sense, a statement with a similar content by the author is only a confirmation of a necessary condition, without any additional information based on Eq. (11).

Finally, trying quantitatively to understand the example, we again met with difficulties to follow the "ultra-hyperbolic calculating mode". Because $r^{2}-\rho^{2} \equiv-4$ (for any $\chi$ ), we obtain in the Eq. (S. (19)) for $\cos \beta$ on S. page 194 a zero, located in denominator, and were thus unable to continue the calculations in order to confirm the results of the author.

\section{SYMMETRIES}

Symmetries play a very important role in quantitative texture analysis and have therefore to be considered with utmost care. The author has, to some extent, taken this into consideration; it is reflected in the $\pm \mathbf{h}_{i}$ solution variants and leads to a reduction of the domain of solution continuation via the condition $G=G^{+} \cap G^{-}$(S.p. 190). But some basic errors appear even at this relatively elementary level.

Over half of the work is devoted to the case $h_{0}=\left(\vartheta_{\mathbf{h}_{0}}=90^{\circ}, \varphi_{\mathbf{h}_{0}}\right)$ for which the statement " $F^{+}=F^{-»}$ is made (S.p. 189 , bottom). This is simply not true as it can be seen, for instance, with Eq. (S. (2)) and (S. (3)) on S. page 186. The cause of such errors is the somewhat lax determination of azimuthal quantities $(0 \leq \phi \leq 2 \pi)$ only via $\tan \phi$ (see the top of S. page 188). In reality, $\phi^{+}$and $\phi^{-}$differ. Therefore, statements like "for every kind of lattice symmetry" (S.p. 190) about the validity of the derived relations are even more surprising, the more 
so as nowhere in the paper can any consideration be found concerning the consequences of the crystal symmetry for the problem described.

Moreover, because "every kind of lattice symmetry" means also the triclinic case, from this view our simplifying (but not changing the validity of the general statements) limitation to this symmetry case in the appendix can be considered as justified.

Up to now in this review, we have deliberately considered only the triclinic case (and ideal pole figures), without the \pm h symmetry appearing due to normal scattering. But the crystal symmetry (point group $\mathcal{G}_{B}$ ) has to be treated in an absolutely equivalent way to the $\pm \mathbf{h}$ symmetry. This means, that the exact relation for the experimental ("reduced") pole figures reads:

$$
\tilde{\boldsymbol{P}}_{\mathbf{h}_{i}}(\mathbf{y})=\sum_{m=1}^{\tilde{N}_{b}} \boldsymbol{P}_{\mathbf{h}_{i_{m}}}(\mathbf{y}) / \tilde{N}_{b}, \quad \mathbf{h}_{i_{m}}=\tilde{g}_{b_{m}} \cdot \mathbf{h}_{i}, \quad \tilde{g}_{b_{m}} \in \tilde{\mathcal{G}_{B}}, \quad \tilde{\mathcal{G}_{B}}=\mathcal{G}_{B} \times C_{i},
$$

where $\mathbf{h}_{\boldsymbol{i}}$, on the left-hand side, is only a name for the whole set of equivalent $\mathbf{h}_{i_{m}}$ (Matthies et al., 1988).

Independently of the fact that, numerically, the $\boldsymbol{P}_{\mathbf{h}_{i m}}(\mathbf{y})$ values for some $m$ can be identical, this is not valid for the gradients in the $h$ space. But these gradients are indispensable for continuations of reasonable solutions. Moreover, the relation $G=G_{\mathbf{h}_{i_{1}}} \cap G_{\mathbf{h}_{i_{2}}} \cdots \cap G_{\mathbf{h}_{i_{\tilde{N}}}}$ should be imposed exactly for the determinable domain. The choice of the "name $\mathbf{h}_{i}{ }^{\text {p for }} \tilde{\boldsymbol{P}}_{\boldsymbol{h}_{i}}(\mathbf{y})$ from the $\mathbf{h}_{i_{m}}$-set is random, but considering a concrete $\mathbf{h}_{i_{m}}$ will get in each case a different continuation of the domain. Therefore, in case of high crystal symmetries, it is quite questionable whether a nonzero $G$ region for continuation of a solution (using the "mixed" variables of the author) even exists at all.

Similar to the above situation of "creating a peak distribution from zero intensities", it should now be shown how, for instance, in the case of a single texture component, four $\left[h_{i}=\left(\begin{array}{lll}1 & 1 & 1\end{array}\right)\right]$ or $24\left[h_{i}=\left(\begin{array}{ll}1 & 23\end{array}\right)\right]$ peaks in the upper pole figure half-sphere can be generated from three peaks in the cubic (001) pole figure. Equation (6), again, has a general validity and "does not know anything" about crystal symmetries. This also applies to any pole figure considered individually, without additionally supplied crystal symmetry related information. This information cannot be introduced by $h \leftrightarrow y$ transformations, but requires 
additional points of input, which should first be shown before statements with so far-reaching consequences can be made as "every kind of ...”.

\section{SINGLE POLE FIGURES}

In order to avoid any risk of misunderstanding with the reader, we would like to underline that it might be possible, in specific cases (very sharp textures, high crystal symmetry, and satisfaction of the necessary MPDS-conditions (Helming, 1992)), to determine an ODF from a single pole figure with a fair degree of approximation. For instance, if only a few nonoverlapping sharp peaks (surrounded by large regions with very small intensities) are present in a cubic (1 111$)$ pole figure. Consequently, using this ODF $f(g)$ it is possible to calculate any other pole figure with the same accuracy. However, this is feasible only by using a "detour via the ODF". It does not require either Eq. (6) or its equivalent formulations as in Eq. (11). But, on the contrary, it needs Eqs. (13) and (3), which are the only relevant ones, and special cases of the structure of the given pole figure.

T.I. Savyolova considers a problem of a totally another type: to calculate in $Y_{1}$ a distribution (designed by her " $h_{1}$ pole figure"), directly from a pole figure $\tilde{P}_{\mathbf{h}_{0}}(\mathbf{y})$ measured in $Y_{0}$, as a continuation of a solution of Eq. (11). Such a "h $h_{1}$ solution" determined by the proposed procedure is not of any practical interest for texture analysis. To name it " $h_{1}$ pole figure" is incorrect. How to construct quite simply (without complicated and rather nontransparent ultra-hyperbolic and $\mathbf{h} \leftrightarrow \mathbf{y}-$ mixing activities) "continuations of solutions" of the same useless type (but also satisfying Eq. (6) and resting only on $P_{\mathrm{h}_{0}}(\mathbf{y})$ data in $Y_{0}$ ), was already mentioned at the end of Section 4 and is described in some more detail in the appendix.

\section{CONCLUSION}

T.I. Savyolova's paper deals with an interesting mathematical problem. However, in our opinion, it does not have any practical consequence for quantitative texture analysis, because the constructed continuations 
of a solution as a rule have nothing to do with real pole figures of interest. Even only trying to go into this direction, there will be difficulties in connection with the discrete character of measurable projection directions $\mathbf{h}_{i}$, with additional restrictions to the formal domain for solution continuations as consequences of a proper treatment of the crystal symmetry as well as of the projection character of pole figures and their definite positivity. It is quite questionable whether a proper treatment of crystal symmetry by $\mathbf{h}, \mathbf{y}$-mixed variables exists at all, these being the basic elements of the author's idea.

In a publication with such spectacular statements, a certain degree of numerical accuracy should be warranted. Moreover, before implementing highly sophisticated mathematical tools, some attention should be paid to simple analogic pictures of the problem, like Fig. 1 that shows right from the beginning the dubious nature of the statements made in the paper in its present form.

In this connection we would like to point out that the computer programs for standard functions have been available to the texture community since 1987 (Matthies et al., 1987), including the scientific environment of the author. They have been specially developed for proper testing procedures with high numerical accuracy and can answer such questions as those treated here (e.g. Figs. 2-5) in a matter of seconds.

\section{References}

Baro, D., Bunge, H.J., Esling, C., Humbert, M., Matthies, S., Muller, J., Van Houtte, P. and Wagner, F. (1982). Metz, unpublished.

Bunge, H.J. (1982). Texture Analysis in Materials Science, London: Butterworths.

Bunge, H.J. and Esling, C. (1979). Texture of Crystalline Solids, 3, 169.

Edmonds, A.R.E. (1957). Angular Momentum in Quantum Mechanics, Princeton Press, New Jersey.

Helming, K. (1992). Textures and Microstructures, 19, 45.

Matthies, S. (1979). Phys. Stat. Solidi (b), 92, k135.

Matthies, S. (1980). Kristall und Technik, 15, 1189.

Matthies, S. (1982). Aktuelle Probleme der quantitativen Texturanalyse, Akademie d. Wiss. d. DDR, ZFK-Rossendorf, ISSN 0138-2950.

Matthies, S., Vinel, G.W. and Helming, K. (1987). Standard Distributions in Texture Analysis, Berlin: Akademie Verlag.

Matthies, S., Wenk, H.R. and Vinel, G.W. (1988). J. Appl. Cryst., 21, 285.

Matthies, S., Helming, K. and Kunze, K. (1990). Phys. Stat. Sol. (b), 157, 489.

Muller, J., Esling, C. and Bunge, H.J. (1981). J. Phys., 42, 161.

Nikolayev, D.I. and Schaeben, H. (1997). NTSA-Conference JINR-Dubna, to be published in Textures and Microstructures. 
Ruer, D. and Baro, R. (1977). J. Appl. Cryst., 10, 458.

Savyolova, T.I. (1982). Rep. of Academy of Sciences USSR, 226, 590.

Savyolova, T.I. (1995). Textures and Microstructures, 23, 185.

Savyolova, T.I (1996). Textures and Microstructures, 25, 183.

Vadon, A. (1981). Thesis, University of Metz.

\section{APPENDIX}

For the formulation of the statements at the level of this reply it is sufficient to consider the ideal case of no crystal symmetry and no $\pm \mathbf{h}$ invariance-related overlaps.

Pole figures - In texture analysis a (ideal) pole figure $P_{\mathrm{h}}(\mathbf{y})$ is defined by Eq. (3) as the two-dimensional projection (projection direction $\mathbf{h}$, two-dimensional projection screen variable $\mathbf{y}$ ) of a given three-dimensional orientation distribution $f(g)$.

Biaxial function - The set of pole figures for the projection directions $h=h_{0}+d h$ in the vicinity of $h_{0}$ can be considered as a part of the biaxial distribution $A(h, y)$ described in the same manner in (3), with

$$
P_{\mathbf{h}_{0}}(\mathbf{y})=A\left(\mathbf{h}_{0}, \mathbf{y}\right) \text {. }
$$

$A(h, y)$ satisfies Eq. (6).

\section{It is said}

(a) Using the data of $P_{\mathbf{h}_{0}}(\mathbf{y})\left(\mathbf{h}_{0}\right.$-section of $\left.A(\mathbf{h}, \mathbf{y})\right)$ for $\mathbf{y} \in \mathbf{Y}_{0}$, it is possible to construct a distribution $B(\mathbf{h}, \mathbf{y})$ ("continuation of a solution"), that also satisfies (6) in the vicinity of $h_{1}\left(h=h_{1}+d h\right)$ and for the y-region $Y_{1}$.

(b) The $h_{1}$ section of this $B(h, y)$ is named " $h_{1}$ pole figure" i.e. it ought to be identical with $P_{\mathrm{h}_{1}}(\mathbf{y})$ in the sense of the projection (3) for a given $f(g)$.

\section{Comment}

The solution of problem (a) is relatively simple. The expression (9) identically satisfies Eq. (6). So $\boldsymbol{P}_{\mathrm{h}_{0}}(\mathbf{y})$ can be represented by the series

$$
P_{\mathbf{h}_{0}}(\mathbf{y})=\sum_{l, n}\left[\frac{4 \pi}{2 l+1} \sum_{m} C_{l}^{m, n} Y_{l, m}^{*}\left(\mathbf{h}_{0}\right)\right] Y_{l, n}(\mathbf{y}),
$$


with the $C_{l}^{m, n}$-coefficients following from the $P_{\mathrm{h}_{0}}(\mathbf{y})$-data. Then $B\left(\mathrm{~h}_{1}, \mathbf{y}\right)$ can be chosen as

$$
B\left(\mathbf{h}_{1}, \mathbf{y}\right)=\sum_{l, n}\left[\frac{4 \pi}{2 l+1} \sum_{m} C_{l}^{m, n} Y_{l, m}^{*}\left(\mathbf{h}_{1}\right)\right] Y_{l, n}(\mathbf{y}) .
$$

From $P_{\mathrm{h}_{0}}(\mathbf{y})$ (with $Y_{0}$ - the complete or incomplete $\mathbf{y}$-region) as a rule the $C_{l}^{m, n}$-coefficients cannot be determined unambiguously. Therefore always an infinity $\left({ }^{k} C_{l}^{m, n} ; k=1,2 \ldots\right)$ of $C_{l}^{m, n}$-sets exists, each of them identically explaining the $P_{\mathrm{h}_{0}}(\mathrm{y})$-data by (A2). Introducing any of these ${ }^{k} C_{l}^{m, n}$-sets into (A3) we get a distribution ${ }^{k} \prod_{h_{1}}(\mathbf{y})$ ("solution") exactly fulfilling Eq. (6) on the whole sphere, if $\Pi$ is considered as biaxial distribution in the vicinity of $h_{1}$. But each of these ${ }^{k} C_{l}^{m, n}$-sets will as a rule lead to different solutions ${ }^{k} \prod_{h_{1}}(\mathbf{y})$, because in spite of

$$
{ }^{k} \prod_{\mathrm{h}_{0}}(\mathbf{y})=P_{\mathrm{h}_{0}}(\mathbf{y})
$$

always an other "projection object" $f_{k}(g)$ will be described:

$$
f_{k}(g)=\sum_{l, m, n}^{k}{ }^{k} C_{l}^{m, n} D_{m, n}^{l}\left(g^{-1}\right),
$$

i.e., to name ${ }^{k} \prod_{h_{1}}(\mathbf{y})$ "pole figure" $P_{\mathbf{h}_{1}}(\mathbf{y})$ (that we will get "measuring" a sample characterized by a certain $f(g)$, or correspondingly calculating $\boldsymbol{P}_{\mathrm{h}_{1}}$ (y) using Eq. (3)) is incorrect. After this any basis for statement (b) (the only moment of practical interest for texture analysis) is completely lost.

\section{Additional Remarks}

The expression (A3) for a "solution" is absolutely free of model. Equation (6) is identically satisfied. Therefore more information cannot in principle be introduced into any scheme of "construction of solutions" by the only application of this equation. This holds also for a $\mathbf{h} \leftrightarrow \mathbf{y}$-mixing variable transformation, leading to the ultra-hyperbolic formulation (11) of Eq. (6). 
Of course, the numerical realization of such a scheme can lead to artificial limitations of the effectively considered " $k$-variants" or to a narrowing of the $Y_{1}$-region, for that the reformulated Eq. (6) will be satisfied. However, any scheme cannot overcome the ambiguity of $f(g)$ (and of the corresponding $P_{\mathrm{h}_{1}}(\mathbf{y})$, that is solely determined by it) if it is resting on some $P_{\mathrm{h}_{0}}(\mathbf{y})$-data only.

The presence of a crystal symmetry, effectively leading to overlaps of (triclinic) ideal pole figures, does not change the situation, because at the end it influences only the pole figure values themselves, but not Eq. (6). The satisfaction of (6) is only a necessary criterion to answer, whether some data for limited $h$ and $\mathbf{y}$ regions can at all be projections (i.e. true pole figures) of any three-dimensional object. An equivalence between Eq. (6) and the projection character of its solutions in the sense of Eq. (3) (sufficient criterion) appears only, if Eq. (6) is satisfied for all $\mathbf{h}$ and $\mathbf{y}$ of a data set. This means, that the solvability of $f(g)$-related problems (like the proper $P_{\mathbf{h}_{1}}(\mathbf{y})$-determination) by Eq. (6) will also need this completeness. And therefore, a $\left(h_{0}, Y_{0}\right) \rightarrow\left(h_{1}, Y_{1}\right)$ coupling of pole figure data (i.e. from limited $h$ regions) using only this equation must inevitably lead to contradictions.

\section{An Old Example}

In principle there is no new knowledge in connection with the analysis of Savyolova's paper, because analogical considerations had been done already at the end of the seventies in connection with the reprojection formula (1), (2) (cf. Matthies, 1980; 1982). But the corresponding example is so transparent, that it is worth remembering it. For simplicity let us take the best situation, i.e. $P_{\mathbf{h}_{0}}(\mathbf{y})$ is known in the complete $y$ region, i.e. if already there are difficulties so much that the more they will arise for an incomplete $Y_{0}$.

The $f_{1}$-part of the $\mathbf{h}_{0}$-related ODF from (2)

$$
f_{1}\left(\mathbf{h}_{0} ; g\right)=P_{\mathbf{h}_{0}}\left(g^{-1} \cdot \mathbf{h}_{0}\right)
$$

is a well-defined orientation distribution (normalized and $\geq 0$ ) in the whole $G$-space and represents an uniform distribution of the $P_{\mathrm{h}_{0}}(\mathbf{y})$ intensities along the corresponding $\left(\mathbf{h}_{\mathbf{0}}, \mathbf{y}\right)$-defined projection threads (cf. Fig. 1). Therefore from this orientation distribution any projections 
$\mathbf{h}$ can be described leading to reasonable solutions ${ }_{1} \prod_{\mathbf{h}}\left(\mathbf{h}_{0} ; \mathbf{y}\right) \geq 0$ in the complete y-region. Because ${ }_{1} \prod_{\mathbf{h}}\left(\mathbf{h}_{0} ; \mathbf{y}\right)$ identically fulfils Eq. (6) we have satisfied all conditions involved in our discussion.

But due to the "blurred" variant of the orientation distribution $f_{1}\left(\mathbf{h}_{0}, g\right)$ (exactly explaining $P_{\mathbf{h}_{0}}(\mathbf{y})$-data) it is obvious, that ${ }_{1} \prod_{\mathbf{h}}\left(\mathbf{h}_{0} ; \mathbf{y}\right)$ as a rule will be different from the true $P_{\mathrm{h}}(\mathbf{y})$ pole figure, that is connected with the original ODF $f(g)$, primary delivering the $P_{\mathbf{h}_{0}}(\mathbf{y})$-data.

Analytically we have

$$
P_{\mathbf{h}_{0}}(\mathbf{y}) \equiv \sum_{l, n} F_{n}^{l}\left(\mathbf{h}_{0}\right) Y_{l, n}(\mathbf{y})
$$

Using this expression $f_{1}\left(\mathbf{h}_{0} ; g\right)$ can be represented by

$$
\begin{aligned}
f_{1}\left(\mathbf{h}_{0} ; g\right) & =\sum_{l, m, n} F_{n}^{l}\left(\mathbf{h}_{0}\right) Y_{l, m}\left(\mathbf{h}_{0}\right) D_{m, n}^{l}\left(g^{-1}\right) \\
& \equiv \sum_{l, m, n} C_{l}^{m, n}\left(\mathbf{h}_{0}\right) D_{m, n}^{l}\left(g^{-1}\right) .
\end{aligned}
$$

Putting these " $C_{l}^{m, n}$-coefficients" into (9) we get the corresponding biaxial distribution $\left(P_{l}(z)-\right.$ Legendre polynominals):

$$
\begin{aligned}
{ }_{1} A\left(\mathbf{h}_{0} ; \mathbf{h}, \mathbf{y}\right) & =\sum_{l, n}\left[\sum_{m} \frac{4 \pi}{2 l+1} F_{n}^{l}\left(\mathbf{h}_{0}\right) Y_{l, m}\left(\mathbf{h}_{0}\right) Y_{l, m}^{*}(\mathbf{h})\right] Y_{l, n}(\mathbf{y}) \\
& \left.=\sum_{l, n} F_{n}^{l}\left(\mathbf{h}_{0}\right) P_{l}\left(\mathbf{h}_{0} \cdot \mathbf{h}\right)\right] Y_{l, n}(\mathbf{y}) \\
& ={ }_{1} \prod_{\mathbf{h}}\left(\mathbf{h}_{0} ; \mathbf{y}\right) .
\end{aligned}
$$

From (A9) and (8) the satisfaction of (6) follows, and due to $P_{l}(1)=1$ (A10) leads for $\mathbf{h}=\mathbf{h}_{\mathbf{0}}$ to the starting pole figure $P_{\mathbf{h}_{0}}(\mathbf{y})(\mathrm{A} 7)$. For $\mathbf{h} \neq \mathbf{h}_{\mathbf{0}}$ the $\mathbf{h}_{0}$-dependence in (A11) will always lead to differences with the "true" projections $\boldsymbol{P}_{\mathrm{h}}(\mathrm{y})$, that do not know anything about our only limited $P_{\mathrm{h}_{0}}(\mathbf{y})$-information on the "true" ODF $f(g)$. 